\title{
Identifying the Superficial Peroneal (Flbular) Nerve for Ultrasound-Guided Ankle Block: A Divisive Issue
}

J Bowness ${ }^{123}$, K Turnbull3, A Taylor2, J Halcrow2, F Chisholm³, O Varsou34, C Grant2 1 Institute of Academic Anaesthesia, University of Dundee, UK

2Department of Anaesthesia, Ninewells Hospital, Dundee, UK

3School of Medicine, University of St Andrews, UK

4 School of Life Sciences, University of Glasgow, UK

james.bowness@nhs.net

\section{7th Annual ESRA Congress, Dublin, 12-15 September 2018}

NHS

Tayside

\section{Aackground |}

- Chin et al showed (2001) a 16\% failure rate for ultrasoundguided ankle block

- We find the region supplied by the superficial peroneal nerve (SPN) to have the highest frequency of incomplete anaesthesia

- We sought to determine whether ultrasound can reliably identify where the SPN pierces deep fascia of the leg, and correlate this to cadaveric findings, to instruct SPN block.

\section{Methods |}

- With ethical approval, we undertook a sonographic assessment of 26 adult volunteers

- The point the SPN pierced deep fascia was measured as a proportion of the distance from the lateral malleolus (LM) to the fibula head (HF)

- The distance anterior/posterior to the LM-HF straight line was measured (figure 1)

- 16 formalin-fixed cadavers were dissected to reveal the SPN, and the same assessment performed (figure 2)
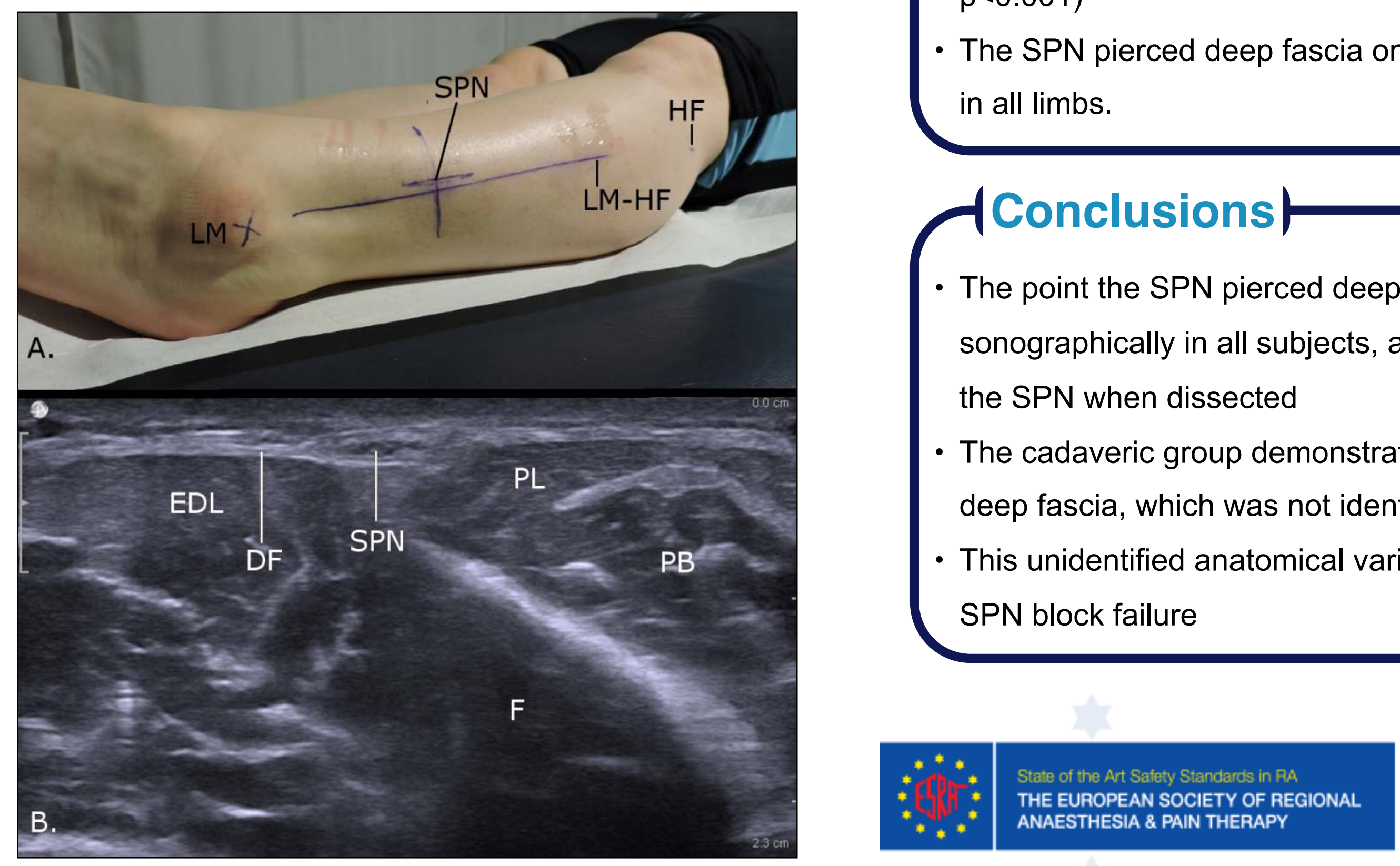

Figure 2. Cadaveric methodology

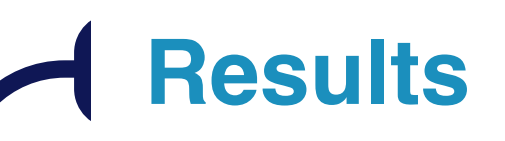

- Sonographically, the SPN was identified piercing deep fascia 0.31 of the total LM-HF distance ( $95 \% \mathrm{Cl} 0.29-0.32)$

- The main SPN trunk pierced at 0.30 in the cadavers $(0.28$ $0.32, \mathrm{t}=-0.391, \mathrm{p}=0.697$ )

- However, the SPN divided before piercing deep fascia in $46.9 \%$ of cadaveric limbs ( $0 \%$ on ultrasound; $t=5.230$, $\mathrm{p}<0.001)$

- The SPN pierced deep fascia on or anterior to the LM-HF line in all limbs.

\section{AConclusions}

- The point the SPN pierced deep fascia was identified sonographically in all subjects, and matched the main trunk of the SPN when dissected

- The cadaveric group demonstrated multiple branches piercing deep fascia, which was not identified on ultrasound

- This unidentified anatomical variation may contribute to USG SPN block failure

Figure 1. Ultrasound methodology

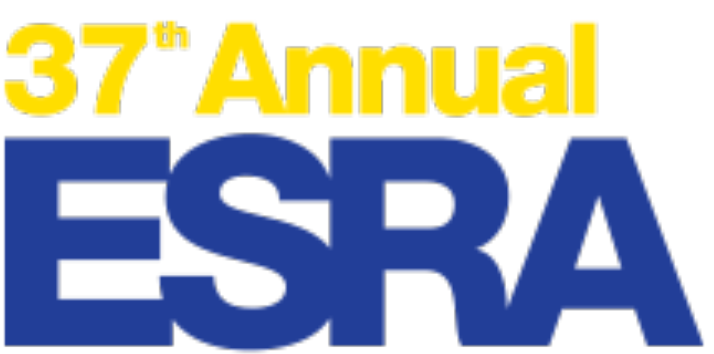

